\title{
Adsorbate-Induced Enhancement of Electrostatic Noncontact Friction
}

\author{
A. I. Volokitin ${ }^{1,2}$ and B. N. J. Persson ${ }^{1}$ \\ ${ }^{1}$ Institut für Festkörperforschung, Forschungszentrum Jülich, D-52425, Germany \\ ${ }^{2}$ Samara State Technical University, 443100 Samara, Russia \\ (Received 1 October 2004; published 3 March 2005)
}

\begin{abstract}
We study the noncontact friction between an atomic force microscope tip and a metal substrate in the presence of bias voltage. The friction is due to energy losses in the sample created by the electromagnetic field from the oscillating charges induced on the tip surface by the bias voltage. We show that the friction can be enhanced by many orders of magnitude if the adsorbate layer can support acoustic vibrations. The theory predicts the magnitude and the distance dependence of friction in good agreement with recent puzzling noncontact friction experiment [B.C. Stipe, H. J. Mamin, T. D. Stowe, T. W. Kenny, and D. Rugar, Phys. Rev. Lett. 87, 096801 (2001).]. We demonstrate that even an isolated adsorbate can produce high enough friction to be measured experimentally.
\end{abstract}

DOI: 10.1103/PhysRevLett.94.086104

A great deal of attention has been devoted to noncontact friction between an atomic force microscope tip and a substrate [1-5]. This is related to the practical importance of noncontact friction for ultrasensitive force detection experiments. The ability to detect small forces is inextricably linked to friction via the fluctuation-dissipation theorem. For example, the detection of single spins by magnetic resonance force microscopy [6], which has been proposed for three-dimensional atomic imaging [7] and quantum computation [8], will require force fluctuations to be reduced to unprecedented levels. In addition, the search for quantum gravitation effects at short length scale [9] and future measurements of the dynamical Casimir forces [10] may eventually be limited by noncontact friction effects. Noncontact friction is also responsible for the friction drag force between two-dimensional quantum wells $[11,12]$.

The noncontact friction always is of electromagnetic origin, but the detailed mechanism is not totally clear, since there are several different mechanisms of energy dissipation connected with the electromagnetic interaction between bodies. First, the fluctuating current density in one body will induce a current density in the other body. The interaction between the fluctuating current and the induced current density gives rise to the well-known long-range attractive van der Waals interaction [13] and van der Waals friction [14-16]. Second, the surfaces of the tip and the sample always have static charges due to inhomogeneities of the surfaces. The presence of inhomogeneous tip-sample electric fields is difficult to avoid, even under the best experimental conditions [3]. The electric field can be easily changed by applying a voltage between the tip and the sample. The friction associated with moving charges will be denoted as the electrostatic friction.

The fluctuating electromagnetic field near the surface of the tip will produce a fluctuating force acting on the tip, resulting in Brownian motion of the cantilever. Thus, just as damping in liquids is intimately connected with random impacts and Brownian motion of small particles, the non-
PACS numbers: 68.35.Af, 68.37.Ps, 73.20.Mf

contact friction is necessarily connected with the random forces that drive the cantilever Brownian motion.

Recently several groups have observed unexpectedly large long-range noncontact friction [1-5]. The friction force $F$ acting on the tip was found to be proportional to the velocity $v, F=\Gamma v$. Stipe et al. [3] observed the noncontact friction effect between a gold surface and a goldcoated cantilever as a function of tip-sample spacing $d$, temperature $T$, and bias voltage $V$. For vibration of the tip parallel to the surface they found $\Gamma(d)=\alpha(T) \times$ $\left(V^{2}+V_{0}^{2}\right) / d^{n}$, where $n=1.3 \pm 0.2$ and $V_{0} \sim 0.2 \mathrm{~V}$ At $295 \mathrm{~K}$, for the spacing $d=100 \AA$ they found $\Gamma=1.5 \times$ $10^{-13} \mathrm{~kg} \mathrm{~s}^{-1}$, An applied voltage of $1 \mathrm{~V}$ resulted in a friction increase of $\Gamma=3 \times 10^{-12} \mathrm{~kg} / \mathrm{s}$ at $300 \mathrm{~K}$ and $d=$ $20 \mathrm{~nm}$.

In a recent Letter, Dorofeyev et al. [1] claim that the noncontact friction effect observed in $[1,2]$ is due to Ohmic losses mediated by the fluctuating electromagnetic field. This result is controversial, however, since the van der Waals friction for good conductors such as copper has been shown [14-17] to be many orders of magnitude smaller than the friction observed by Dorofeyev et al. Realizing that the van der Waals friction between good conductors is too small to explain the experimental data, in [18] it was proposed that the van der Waals friction may be strongly enhanced between the high resistivity mica substrate and the silica tip. However, the mica substrate and the silica tip were coated by gold films thick enough to completely screen the electrodynamic interaction between underlying dielectrics. The large friction obtained in [18] is due to the extremely small conductivity of mica $[\sigma \sim$ $\left.10^{-8}(\Omega \mathrm{m})^{-1}\right]$.

At small separation $d \sim 1 \mathrm{~nm}$ resonant photon tunneling between the adsorbate vibrational modes on the surfaces of the tip and the sample can enhance the friction by 7 orders of magnitude in comparison with the good conductors surfaces $[15,16]$. However, the distance dependence $\left(\sim 1 / d^{6}\right)$ is stronger than observed experimentally. 
Recently, a theory of noncontact friction was suggested where the friction arises from Ohmic losses associated with the electromagnetic field created by moving charges induced by the bias voltage [19]. In the case of a spherical tip this theory predicts the same weak distance dependence of the friction as observed in the experiment, but the magnitude of the friction is many orders of magnitude smaller than the experimental value. At present there is no theory which can explain satisfactorily both the magnitude and the distance dependence of friction observed in [3].

In this Letter we present a novel explanation of the puzzling experimental data reported in [3]. We suggest that the large long-range noncontact friction is due to the electromagnetic interaction of the moving charges, induced on the surface of the tip by the bias voltage, with acoustic vibrations in an adsorbate layer on the surface of the sample. In particular, for the $\mathrm{Cs} / \mathrm{Cu}(001)$ system the experiment suggests the existence of an acoustical film mode even for the very dilute phase $(\theta=0.08)$ [20]. The negligible value of the substrate corrugation for $\mathrm{Cs} / \mathrm{Cu}(001)$ explains why no preferential adsorption site has ever been observed for this system.

We begin by considering a model in which the tip of a metallic cantilever of length $L$ is a section of a cylindrical surface with radius of curvature $R$ (see Fig. 1). The cantilever is perpendicular to a flat sample surface, which occupies the $x y$ plane, with the $z$ axis pointing into the sample. The tip displacement $\mathbf{u}(t)=\hat{x} u_{0} e^{-i \omega t}$ is assumed to be parallel to the surface (along the $x$ axis), which will be a good approximation when the oscillation amplitudes $u_{0}$ are sufficiently small. The cantilever width $b$, i.e., the size in the direction perpendicular to the $x z$ plane, is taken to be much larger than the thickness $c(b \gg c)$. In the case of a cylindrical tip geometry, the electric field induced by the bias voltage is the same as that which would be produced in the vacuum region by two charged wires passing through points at $z= \pm d_{1}= \pm \sqrt{2 d R+\bar{d}^{2}}$, where $d$ is the separation between the cylinder and the sample surface [21]. The wires have charges $\pm Q$ per unit length, $Q=C V$, where $V$ is the bias voltage, and $C^{-1}=2 \ln [(d+R+$ $\left.d_{1}\right) / R$ ]. The vibration of the tip will produce an oscillating electromagnetic field which is the same as that which would be produced in the vacuum region from an oscillat-

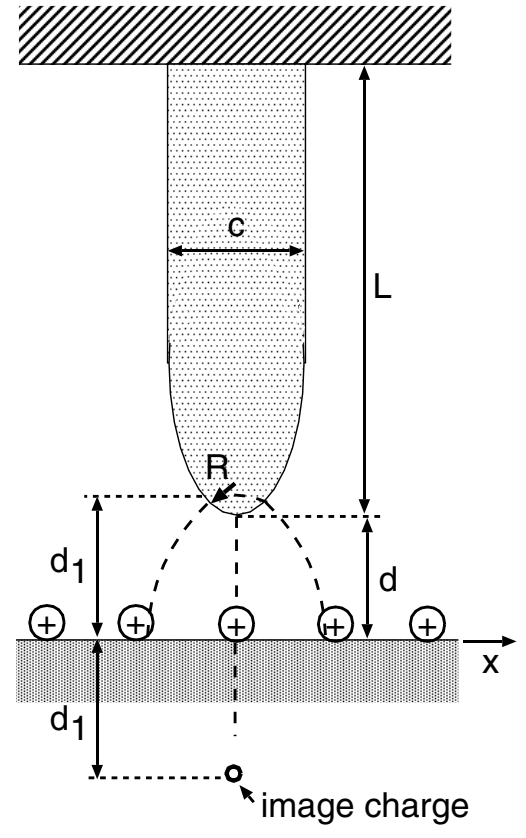

FIG. 1. Scheme of the tip-sample system. The tip shape is characterized by its length $L$ and the tip radius of curvature $R$.

ing charged wire located at $z=d_{1}$. The energy dissipation in the sample induced by the electromagnetic field of the oscillating wire is determined by integrating Poynting's vector over the sample surface. Taking into account that the energy dissipation per unit time must be equal to $2 \omega^{2} \Gamma\left|u_{0}\right|^{2}$, we get the following formula for friction coefficient for motion of the cylindrical tip parallel to sample surface:

$$
\Gamma_{\|}=\lim _{\omega \rightarrow 0} 2 Q^{2} b \int_{0}^{\infty} d q q e^{-2 q d_{1}} \frac{\operatorname{Im} R_{p}(\omega, q)}{\omega}
$$

where $R_{p}$ is the reflection coefficient for $p$-polarized electromagnetic waves.

The reflection coefficient $R_{p}$, which takes into account the contribution from an adsorbate layer, can be obtained using an approach which was proposed in [22]. Using this approach we get

$$
R_{p}=\frac{1-s / q \epsilon+4 \pi n_{a}\left[s \alpha_{\|} / \epsilon+q \alpha_{\perp}\right]-q a\left(1-4 \pi n_{a} q \alpha_{\|}\right)}{1+s / q \epsilon+4 \pi n_{a}\left[s \alpha_{\|} / \epsilon-q \alpha_{\perp}\right]+q a\left(1+4 \pi n_{a} q \alpha_{\|}\right)},
$$

where $s=\sqrt{q^{2}-(\omega / c)^{2}} \epsilon, \alpha_{\|}$and $\alpha_{\perp}$ are the polarizabilities of adsorbates in a direction parallel and normal to the surface, respectively. $\epsilon=1+4 \pi i \sigma / \omega$ is the bulk dielectric function, where $\sigma$ is a conductivity, and $n_{a}$ is the concentration of adsorbates. In comparison with the expression obtained in [22], Eq. (2) takes into account that the centers of the adsorbates are located at distance $a$ away from image plane of the metal. Although this gives corrections of the order $q a \ll 1$ to the reflection amplitude, for parallel adsorbate vibrations on the good conductors (when $\epsilon \gg 1$ ), they give the most important contribution to the energy dissipation. For clean surfaces $n_{a}=0$, and in this case formula (2) reduces to the well-known Fresnel formula. In this case, for $R \gg d$, Eq. (1) gives a formula 
which was obtained recently in [19] using a less general approach:

$$
\Gamma_{c l}^{c}=\frac{b V^{2}}{2^{6} \pi \sigma d^{2}} .
$$

With $b=7 \times 10^{-6} \mathrm{~m}$ and $\sigma=4 \times 10^{17} \mathrm{~s}^{-1}$ for gold at $300 \mathrm{~K}$, and with $d=20 \mathrm{~nm}$ and $V=1 \mathrm{~V}$ this formula gives a friction which is 8 orders of magnitude smaller than observed experimentally [3].

Let us now consider ions with charge $e^{*}$ adsorbed on the sample. The polarizability for ion vibration normal and parallel to the surface is given by

$$
\alpha_{\perp(\|)}=\frac{e^{* 2}}{M\left(\omega_{\perp(\|)}^{2}-\omega^{2}-i \omega \eta_{\perp(\|)}\right)},
$$

where $\omega_{\perp(\|)}$ is the frequency of the normal (parallel) adsorbate vibration, $\eta_{\perp(\|)}$ is the corresponding damping constant, and $M$ is the adsorbate mass. A particular interesting case is $\mathrm{Cs}$ adsorbed on $\mathrm{Cu}(100)$. At $\theta \approx 0.1$ the $\mathrm{Cs}$ film has an acoustical branch with adsorbate vibrations parallel to the surface. This means that for such vibrations $\omega_{\|} \approx 0$. In this case the main contribution to the friction comes from vibrations parallel to the surface, and the imaginary part of the reflection amplitude is given by

$$
\operatorname{Im} R \approx \frac{2 \omega \eta q a \omega_{q}^{2}}{\left(\omega^{2}-\omega_{q}^{2}\right)^{2}+\omega^{2} \eta^{2}},
$$

where $\omega_{q}^{2}=4 \pi n_{a} e^{* 2} a q^{2} / M$. Using (5) in (1) for $R \gg d$ we get

$$
\Gamma_{a d}^{c}=\frac{b \eta M R^{1 / 2} V^{2}}{2^{7 / 2} d^{3 / 2} \pi n_{a} e^{* 2}} .
$$

This friction exhibits the same distance dependence as is observed experimentally [3]. For isolated $\mathrm{Cs}$ on $\mathrm{Cu}(100)$ the damping parameter $\eta$ has both phononic and electronic contributions. The friction due to one-phonon processes gives $\eta_{p h}=2.6 \times 10^{8} \mathrm{~s}^{-1}$ [20]. However, the existence of the acoustic branch in the adsorbed layer means that the adsorbate layer is incommensurate with the substrate. In this case emission of the bulk phonons gives a vanishing contribution to the damping of parallel vibrations in the adsorbed layer because the adatoms do not "see" the corrugation of the substrate potential.

For $\mathrm{Cs}$ adsorbed on $\mathrm{Cu}(100)$ the adsorption height $a=$ $2.94 \AA$ and at coverage $\theta \approx 0.1$ the dipole moment $\mu \approx$ $4 D$ [20]; thus the ion charge $e^{*}=\mu / a \approx 0.28 e$. In this case the theory of the electronic friction for ionic bond [23,24] gives $\eta_{\| \text {ion }}=3 \times 10^{7} \mathrm{~s}^{-1}$. This is a rather small damping constant. A much larger contribution comes from electronic friction due to covalent bond which is given by $[23,25]$

$$
\eta_{\| \mathrm{cov}}=\frac{2}{\pi \hbar} \xi \frac{m_{e}}{M} \epsilon_{F} \sin ^{2} \frac{\pi e^{*}}{e}
$$

where for $\mathrm{Cs}$ adsorbed on $\mathrm{Cu}(100)$ the parameter $\xi \approx 0.17$, $m_{e}$ is the electron mass, and $\boldsymbol{\epsilon}_{F}$ is the Fermi energy. With $\epsilon_{F}=7 \mathrm{eV}, \quad e^{*} / e=0.28, \quad$ Eq. (7) gives $\eta_{\| \mathrm{cov}}=$ $3 \times 10^{9} \mathrm{~s}^{-1}$ and with $n_{a}=10^{18} \mathrm{~m}^{-2} \quad(\theta \approx 0.1), R=$ $10^{3} \mathrm{~nm}$, and with the same other parameters as above at $d=20 \mathrm{~nm}$ Eq. (6) gives $\Gamma=2.7 \times 10^{-13}$, which is 7 orders of magnitude larger than for the clean surface and only 1 order of magnitude smaller than observed experimentally [3]. Because of the similarities of $\mathrm{Cu}$ and $\mathrm{Au}$ surfaces the same estimations will be valid also for the Au surface. However, the electronic friction predicts weaker temperature dependence than in the experiment, where the friction at $300 \mathrm{~K}$ for $d=20 \mathrm{~nm}$ is approximately 6 times larger than at $77 \mathrm{~K}$ regardless of voltage.

Defects of the adsorbed layer such as atomic steps, impurity atoms, and other imperfections, and also the anharmonicity, will also contribute to vibrational relaxation in the adsorbed layer. The anharmonicity and vacancies give a temperature dependent contribution to the damping parameter $\eta$. The vacancies give exponential dependence on the temperature, and anharmonicity gives contributions $\sim T$ for three-phonon processes and $\sim T^{2}$ for four-phonon processes. Further experimental and theoretical studies will be required to fully elucidate the origin of the phonon lifetime in the adsorbed layer.

In the case of a spherical tip geometry the electric field induced by the bias voltage is approximately the same as that which would be produced in the vacuum region between two point charges $\pm Q= \pm C V$ located at

$$
z= \pm d_{1}= \pm \sqrt{3 R d / 2+\sqrt{(3 R d / 2)^{2}+R d^{3}+d^{4}}}
$$

where $C=\left(d_{1}^{2}-d^{2}\right) / 2 d$. It can been shown that the electrostatic force between the tip and the metal surface within this approximation agrees very well with the exact expression for a sphere above metal surface [26]. The vibrations of the tip will produce an oscillating electromagnetic field, which in the vacuum region coincides with the electromagnetic field of an oscillating point charge. The friction coefficient for a point charge moving parallel to the surface due to the electromagnetic energy losses inside the sample determined by [27]

$$
\Gamma_{\|}=\lim _{\omega \rightarrow 0} \frac{Q^{2}}{2} \int_{0}^{\infty} d q q^{2} e^{-2 q d_{1}} \frac{\operatorname{Im} R_{p}(\omega, q)}{\omega} .
$$

For motion normal to the surface, $\Gamma_{\perp}=2 \Gamma_{\|}$. For a clean sample surface and for $R \gg d$ from Eq. (9) we get

$$
\Gamma_{c l}^{s}=\frac{3^{1 / 2} R^{1 / 2} V^{2}}{2^{7} d^{3 / 2} \pi \sigma} .
$$

This expression is only a factor of 1.6 smaller than that obtained independently in [19] using a less general approach. For the same parameters as above and at $d=$ $20 \mathrm{~nm}$, the friction for a spherical tip is 2 orders of magnitude smaller than for the cylindrical tip. For a spherical tip, 
when the sample surface is covered by adsorbates, from Eqs. (5) and (9) for $R \gg d$ we get the contribution to the friction from adsorbates

$$
\Gamma_{a d}^{s}=\frac{3 R M \eta V^{2}}{2^{6} d \pi n_{a} e^{* 2}} .
$$

This friction at $d=20 \mathrm{~nm}$ is also 2 orders of magnitude smaller than for the cylindrical tip.

We now show that the atomic force microscope can be used to study the vibrational dynamic of isolated adsorbates. Let us calculate the friction acting on the tip due to the interaction of the tip with vibrations of an isolated adsorbate. Let us assume that the tip is in a top position relative to an adsorbate. In this case, due to vibrations of the ion parallel to the surface, a fluctuating force will act on a spherical tip in the direction normal to the surface

$$
F_{z}^{f}=\frac{12 Q \mu u_{a \|}^{2}}{d_{1}^{5}}
$$

where $u_{a \|}$ is the displacement coordinate for vibrations of the ion parallel to the surface, $\mu$ is the dipole moment of the isolated ion. Accordingly to the Kubo formula, the friction coefficient can be expressed through the forceforce correlation function [28]

$$
\Gamma_{\perp}=\left(k_{B} T\right)^{-1} \operatorname{Re} \int_{0}^{\infty} d t\left\langle F_{z}^{f}(t) F_{z}^{f}(0)\right\rangle,
$$

where $\langle\cdots\rangle$ stands for thermal equilibrium average. Using Eqs. (12) and (13) for $d \ll R$ we get

$$
\Gamma_{\perp}=1.3 \frac{\mu^{2} k_{B} T V^{2}}{M^{2} \omega_{\|}^{4} d^{5} R^{3} \eta} .
$$

For the $\mathrm{Cs} / \mathrm{Cu}(100)$ system $\mu=7.5 \mathrm{D}, \omega_{\|}=0.9 \times$ $10^{12} \mathrm{~s}^{-1}$, and $\eta=4.6 \times 10^{9} \mathrm{~s}^{-1}$ [20]. For a sharp tip with $R=2 d=1 \mathrm{~nm}, V=1 \mathrm{~V}$, and $T=300 \mathrm{~K}$ we get friction $\Gamma_{\|}=7.1 \times 10^{-13} \mathrm{~kg} / \mathrm{s}$. This friction is so large that it can be measured with present state-of-the-art equipment. Note that the friction is characterized by a very strong distance dependence, and this can be used for the determination of the ion position.

In this Letter, we have shown that the "electrostatic friction" can be enhanced by several orders of magnitude when the surface of the metal sample is covered by an adsorbed layer with an acoustic branch of vibrations parallel to the surface. Our calculations predict a noncontact friction with similar magnitude, distance, and bias voltage dependence as observed in a recent atomic force microscope study [3]. We have demonstrated that even an isolated adsorbate can produce high enough friction which can be measured experimentally. These results should have broad application in noncontact friction microscopy and in the design of new tools for studying adsorbate vibrational dynamics.
A. I. V. acknowledges financial support from the Russian Foudation for Basic Research (Grant No. 04-02-17606). B.N.J.P. and A.I.V. acknowledge support from the European Union Smart QuasiCrystals project.

[1] I. Dorofeyev, H. Fuchs, G. Wenning, and B. Gotsmann, Phys. Rev. Lett. 83, 2402 (1999).

[2] B. Gotsmann and H. Fuchs, Phys. Rev. Lett. 86, 2597 (2001).

[3] B. C. Stipe, H. J. Mamin, T. D. Stowe, T. W. Kenny, and D. Rugar, Phys. Rev. Lett. 87, 096801 (2001).

[4] H. J. Mamin and D. Rugar, Appl. Phys. Lett. 79, 3358 (2001).

[5] P. M. Hoffmann, S. Jeffery, J. B. Pethica, H. Özgür Özer, and A. Oral, Phys. Rev. Lett. 87, 265502 (2001).

[6] D. Rugar, R. Budakian, H. J. Mamin, and B. W. Chui, Nature (London) 430, 329 (2004).

[7] J. A. Sidles et al., Rev. Mod. Phys. 67, 249 (1995).

[8] G. P. Berman, G. D. Doolen, P.C. Hammel, and V.I. Tsifrinovich, Phys. Rev. B 61, 14694 (2000).

[9] N. Arkani-Hamed, S. Dimopoulos, and G. Dvali, Phys. Lett. B 429, 263 (1998); Sci. Am. 283, No. 2, 62 (2000).

[10] U. Mohideen and A. Roy, Phys. Rev. Lett. 81, 4549 (1998).

[11] T. J. Gramila, J.P. Eisenstein, A. H. MacDonald, L. N. Pfeiffer, and K.W. West, Phys. Rev. Lett. 66, 1216 (1991); Surf. Sci. 263, 446 (1992).

[12] U. Sivan, P. M. Solomon, and H. Shtrikman, Phys. Rev. Lett. 68, 1196 (1992).

[13] I. E. Dzyaloshinskii, E. M. Lifshitz, and L. P. Pitaevskii, Adv. Phys. 10, 165 (1961).

[14] A. I. Volokitin and B. N. J. Persson, J. Phys. Condens. Matter 11, 345 (1999).

[15] A. I. Volokitin and B. N. J. Persson, Phys. Rev. Lett. 91, 106101 (2003).

[16] A. I. Volokitin and B. N. J. Persson, Phys. Rev. B 68, 155420 (2003).

[17] B. N. J. Persson and A. I. Volokitin, Phys. Rev. Lett. 84, 3504 (2000).

[18] J.R. Zurita-Sánchez, J.-J.Greffet, and L. Novotny, Phys. Rev. A 69, 022902 (2004).

[19] A. A. Chumak, P. W. Milonni, and G. P. Berman, Phys. Rev. B 70, 085407 (2004).

[20] P. Senet, J. P. Toennies, and G. Witte, Chem. Phys. Lett. 299, 389 (1999).

[21] L.D. Landau and E. M. Lifshitz, Electrodynamics of Continious Media (Pergamon, London, 1960).

[22] D. C. Langreth, Phys. Rev. B 39, 10020 (1989).

[23] B. N. J. Persson, Phys. Rev. B 44, 3277 (1991).

[24] A. Liebsch, Phys. Rev. B 55, 13263 (1997).

[25] A. I. Volokitin, O. M. Braun, and V.M. Yakovlev, Surf. Sci. 172, 31 (1986).

[26] S. Hudlet, M.S. Jean, C. Guthmann, and J. Berger, Eur. Phys. J. B 2, 5 (1998).

[27] B. N. J. Persson and W. L. Schaich, J. Phys. C 14, 5583 (1981).

[28] E. G. d'Agliano, P. Kumar, W. Schaich, and H. Suhl, Phys. Rev. B 11, 2122 (1975). 